\title{
Iterative Turbo Beamforming for OFDM based Hybrid Terrestrial-Satellite Mobile System
}

\author{
Ammar H. Khan, Muhammad A. Imran and Barry G. Evans \\ Centre for Communication Systems Research (CCSR) \\ University of Surrey, Guildford GU2 7XH UK \\ Email: A.H.Khan@surrey.ac.uk
}

\begin{abstract}
In the context of Orthogonal Frequency Division Multiplexing (OFDM) based systems, pilot based Beamforming (BF) exhibits a high degree of sensitivity to the pilot sub-carriers. Increasing the number of reference pilots significantly improves BF performance as well as system performance. However, this increase comes at the cost of data throughput which inevitably shrinks due to transmission of additional pilots. Hence an approach where reference signals available to the BF process can be increased without transmitting additional pilots can exhibit superior system performance without compromising throughput. Thus, in this paper we present a novel three-stage Iterative Turbo Beamforming (ITBF) algorithm for an OFDM based Hybrid Terrestrial-Satellite Mobile System which utilises both pilots and data to perform interference mitigation. Data sub-carriers are utilised as virtual reference signals in the BF process. Results show that when compared to non-iterative conventional BF, the proposed ITBF exhibits Bit Error Rate Gain (BERG) of up to $2.5 \mathrm{~dB}$ with only one iteration.
\end{abstract}

\section{Introduction}

Advanced array processing combined with OFDM technology forms a comprehensive solution for future high capacity communication networks. Along with provisioning of high data rates, future network services will also need to have global presence to ensure success. In the light of this, we proposed an OFDM based Hybrid Terrestrial-Satellite Mobile System (HTSMS) [1] where users in urban and rural areas are served by satellite and terrestrial cellular Base Stations (BTSs) in an integrated and transparent fashion. This is hybrid in the sense that the BTSs provide service to mobile customers in urban areas while the satellite network serves users in rural areas. In the proposed HTSMS, terrestrial and satellite networks reuse 
the spectrum dedicated to each other resulting in an increase of the overall capacity. Furthermore, to enable use of similar mobile terminals within both networks, the service provisioning by the two networks would be transparent to the end user. However due to frequency reuse, Co-Channel Interference (CCI) is induced by the terrestrial users to the satellite. To mitigate this CCI, we employ Least Mean Squares (LMS) adaptive BF at the satellite [1]. Other variants of LMS can also be adopted such as NLMS [2, 3] and VSS-LMS [4] which provide better convergence. We have implemented NLMS and VSS-LMS for a satellite scenario in our previous work [5] but here we focus on Optimised-LMS.

In terms of interference mitigation, BF performance is greatly influenced by the design of pilots [6]. In light of this, we proposed a preamble based beamformer $[7,5]$ and show that $\mathrm{BF}$ convergence performance is extremely sensitive to OFDM reference signal structure. Specifically, we form pilots as preambles during BF convergence phase which results in significant improvements, both in terms of BF as well as in the overall system performance. Therefore if number of pilot sub-carriers in an OFDM system are increased throughout the transmission, reference signals for the BF process increase which will result in enhanced interference mitigation, but achieve this at the cost of data throughput. However we also receive data in conjunction with the pilots in an OFDM receiver. If this data can be used along with pilot sub-carriers to perform BF, this can enable superior performance without sacrifice of data throughput. Thus, we propose a novel iterative beamformer which uses both pilots and data for BF. Depending on the reliability of the data received, we formulate a data plus pilot driven BF which exhibits significant gains in terms of system performance.

\section{Related Work and Algorithm Formulation}

The innovative iterative turbo receiver proposed by Berrou et al. [8] demonstrated that turbo codes exhibit near Shannon capacity in an AWGN channel. Thereafter, the iterative turbo receiver design has not been restricted to only decoding of turbo codes. The turbo principle has been successfully applied to other communication problems such as channel estimation, joint source and channel coding, synchronization and multi-user detection. Turbo receiver design also finds use in interference mitigation applications such as BF. Sellathurai and Haykin in [9] proposed an iterative beamformer for multi-transmit, multireceive wireless communication systems. They present a maximum a posteriori probability (MAP) decoder based iterative receiver in conjunction with a soft iterative interference canceller that employs turbo-like processing for Bell Labs Layered Space-Time (BLAST) architecture [10]. Using similar turbo principles, authors in [11, 12] present iterative BF and multi-user detection for CDMA based systems. Hunziker et al in [13] propose a Sample Matrix Inversion (SMI) working in conjunction with MAP, working iteratively to mitigate system interference. By employing the iterative BF, authors show effective CCI cancellation in wireless ad-hoc networks with uncoordinated channel access and propose it as an alternative to collision avoidance protocols. The authors extended their work for SIMO-OFDM wireless ad-hoc systems in [14] and showed effective CCI mitigation using a similar SMI based iterative beamformer. Authors in [15] propose an iterative symbol-level transmit and receive beamformer with the objective of SINR maximisation, whereas authors in [16] propose a smooth beamformer 
based on orthogonal iterations across sub-carriers. Specific to OFDM, recently Zhao et al. [17] proposed a turbo based channel estimator which aims to reduce ICI induced in OFDM systems due to users mobility. Most of the aforementioned iterative receiver designs for BF are non-OFDM systems. Work in [14] is based on OFDM but is related to ad-hoc networks and focuses on collision avoidance. Moreover, authors use SMI based BF which is complex as compared to LMS. Work in [17] is again based on OFDM but focuses on ICI mitigation through enhanced turbo channel estimation and does not involve $\mathrm{BF}$ or $\mathrm{CCI}$ mitigation.

In this paper we propose a novel symbol-level LMS based iterative beamformer that uses turbo processing approach to mitigate CCI for the HTSMS uplink scenario. Compared to conventional non-iterative BF methods, the proposed beamformer uses both pilots and soft decoded data information with the turbo principle to enhance interference mitigation. As compared to the aforementioned iterative approaches, the proposed technique is a three-stage OFDM based LMS beamformer which improves system performance with respect to the soft data input. More specifically, the beamformer is based on improving the a priori information of the soft decoded data and the pilots by adapting BF weights according to the respective levels of reliability. The turbo-like procedure enhances BF performance which in turn leads to improved system performance. In terms of the BF algorithm, we opt for the less complex Pre-FFT solution which employs one complex BF weight per antenna element updated at each recursion. However the Pre-FFT saves computational resources at the cost of being sub-optimal. Specifically, it translates to narrowband BF in a wideband OFDM system scenario. The impact of this trade-off would be more prominent when number of sub-carriers in OFDM symbols are large, specifically in sever channel conditions. In that case, Pre-FFT may no longer be a viable solution. An alternative approach is spatial signal processing of individual sub-carriers in the frequency domain which is known to provide the optimum performance [18] at the expense of higher complexity. Hence for an OFDM system, Post-FFT or sub-carrier based BF would provide a far superior performance in terms of convergence and error rate while compromising heavily on the complexity. For instance, if a $N$ sub-carrier OFDM system with $S$ antenna elements undergoes a total of $L$ BF recursions, then Pre-FFT has complexity of the order of $S \times L$ where as the Post-FFT has $N \times S \times L$. Moreover, Post-FFT has scalability issues with regards to number of sub-carriers, with complexity of the BF mechanism being direct proportional to number of OFDM sub-carriers. To reduce complexity of the Post-FFT approach, several solutions have been proposed such as sub-carrier clustering based BF [19] and Multi-Stage BF [20].

The following notations will be used throughout the paper. $\mathbf{A}$ and $\tilde{\mathbf{A}}$ denote a matrix in the time and frequency-domain respectively, whereas a represents a vector. $[\mathbf{A}]_{n, m}$ represents an element at the $n^{\text {th }}$ row and $m^{\text {th }}$ column of $\mathbf{A}$.

\section{System Model}

HTSMS is envisioned to offer global coverage by operating terrestrial and satellite networks in an integrated framework. Fig. 1 depicts the devised system scenario under study with a hybrid framework. We focus on the mitigation of CCI induced by terrestrial mobile users from the perspective of a Geostationary (GEO) satellite. With respect to the system, a link between 


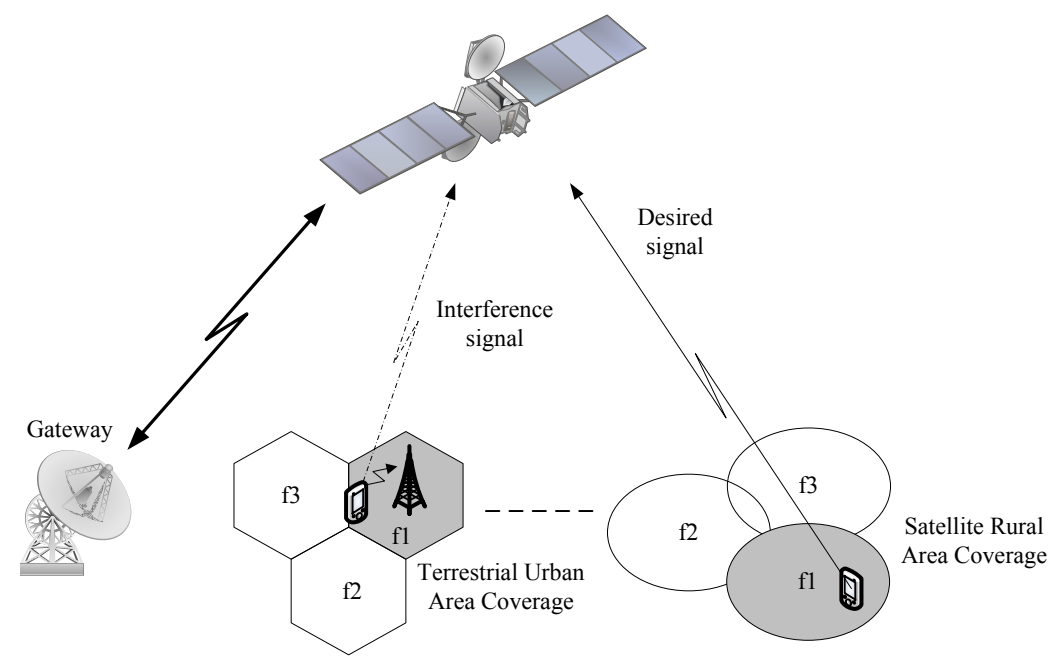

Figure 1. Hybrid Terrestrial-Satellite Mobile System scenario

mobile and satellite is modelled as Single Input Multiple Output (SIMO). Total $J$ users are considered in the system, with one desired user denoted as $d$ being served by the satellite while the rest being served by terrestrial BTSs. After the signal passes through the wireless channel, BF is applied at the satellite end to mitigate interference induced by terrestrial users. The interference model corresponding to the desired and interference signals and geometry of their respective Direction-of-Arrival (DOA) is illustrated in Fig. 2. Due to onboard implementation constraints and less severe satellite channel environment, we employ less complex time-domain BF (Pre-FFT). We focus on interference mitigation performance while assuming the Radio Access Network (RAN) employs an adequate Radio Resource Management (RRM) system which is able to correct time and frequency errors. OFDM as being a multi-carrier system is susceptible to Carrier Frequency Offsets (CFO) [21] which results into loss in sub-carrier orthogonality, hence rising the level of Inter Carrier Interference (ICI). As our work focuses on CCI mitigation, we assume the RAN employs appropriate CFO estimation and recovery [22]. However as CFO can severely effect the system performance, in this paper we also investigate the impact of ICI arising due to CFO on system's throughput.

\subsection{BICM-OFDM Model for HTSMS}

Transceiver architecture for a BICM-OFDM HTSMS is presented in Fig. 3 and this will be referred to throughout the paper to follow the information flow in the system. At the transmitter end of the $j^{\text {th }}$ user $(j=1, \ldots, J)$, information bits $\{\mathbf{o}\}$ are generated and encoded into $\{\mathbf{t}\}$ and then interleaved into $\{\mathbf{c}\}$. The Interleaved bits are then mapped into QPSK complex symbols and Serial-to-Parallel (S/P) converted to $\left\{\widetilde{\mathbf{x}}^{q}\right\}$. Pilots $\left\{\widetilde{\mathbf{x}}^{p}\right\}$ are interspersed into data sequence $\left\{\widetilde{\mathbf{x}}^{q}\right\}$ at known pilot sub-carriers $\{\mathcal{I}\}$. The process outputs $N$ sub-carrier OFDM symbol that can be expressed as:

$$
\widetilde{\mathbf{x}}_{j}=\left[\widetilde{x}_{j}(0), \widetilde{x}_{j}(1), \ldots, \widetilde{x}_{j}(N-1)\right]^{T} .
$$




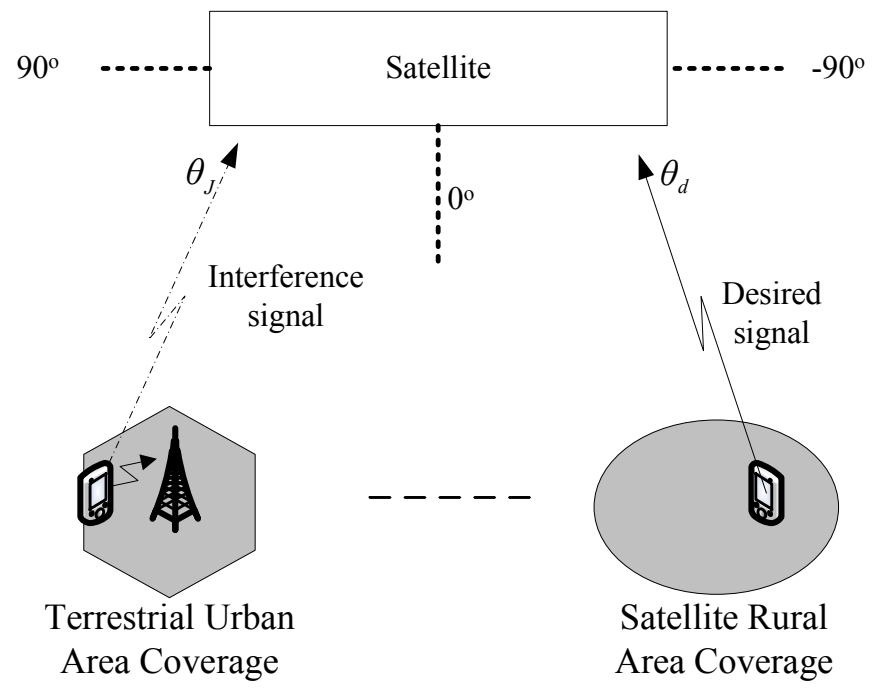

Figure 2. Interference model

For the sake of brevity, we drop the subscript $j$ that indicates user indexing. After formation of OFDM symbol, $\widetilde{\mathbf{x}}$ is converted to the time-domain by a $N$-point IFFT which is given by:

$$
\mathbf{x}=\mathbf{F}^{H} \widetilde{\mathbf{x}}
$$

where

$$
\mathbf{F}=\left[\begin{array}{cccc}
1 & 1 & \cdots & 1 \\
1 & e^{-j 2 \pi(1)(1) / N} & \cdots & e^{-j 2 \pi(1)(N-1) / N} \\
\vdots & \vdots & \ddots & \vdots \\
1 & e^{-j 2 \pi(N-1)(1) / N} & \cdots & e^{-j 2 \pi(N-1)(N-1) / N}
\end{array}\right]
$$

At the start of the OFDM symbol, a CP of length $G$ is appended and the output $\overline{\mathbf{x}}=[x(-G), x(-G+1), \ldots, x(N-1)]^{T}$ is serially transmitted. At the satellite end, we model a Uniform Linear Array (ULA) of antenna elements whose output after CP removal for the $l^{\text {th }}$ OFDM symbol $(l=1, \ldots, L)$ for all the users can be presented as:

$$
\mathbf{V}=\mathbf{A} \mathbf{Y}^{H}+\mathbf{B}
$$

where $[\mathbf{Y}]_{s, j}$ represents the received signal at the $s^{t h}$ antenna element for the $j^{t h}$ user and $s=1, \ldots, S$ is the indexing for array elements in the ULA. $[\mathbf{B}]_{s, n}$ and $[\mathbf{V}]_{s, n}$ represent the i.i.d complex Gaussian noise $\sim \mathcal{C N}\left(0, \sigma^{2}\right)$ and ULA output at the $s^{t h}$ antenna element and $n^{t h}$ sub-carrier respectively. A presents the ULA response, where $[\mathbf{A}]_{s, j}$ can be presented as:

$$
a(s, j)=e^{\left(-j 2 \pi(s-1) d_{a} \sin \left(\theta_{j}\right) / \lambda\right)}
$$




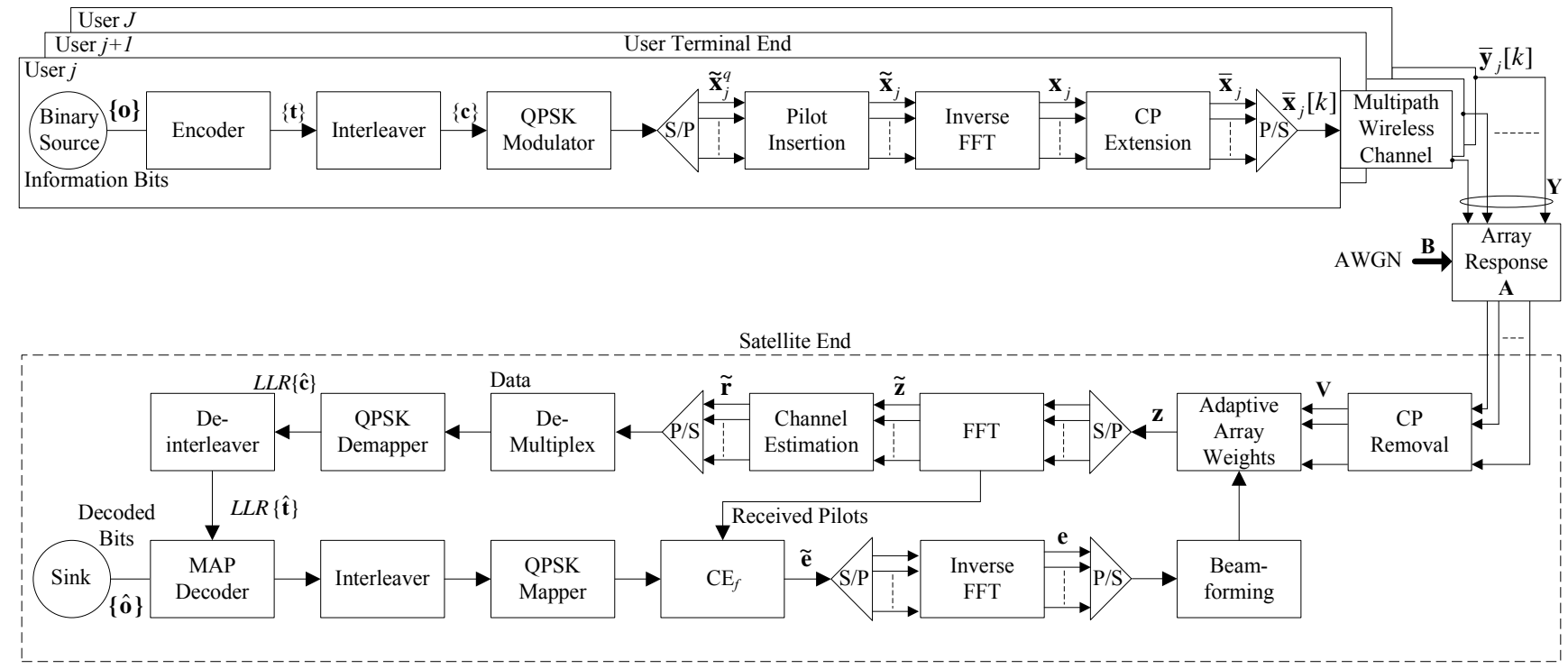

Figure 3. BICM-OFDM system for HTSMS with ITBF

In (5), $d_{a}=\lambda / 2$ is the inter-antenna element spacing, $\theta_{j}$ is the DOA of the $j^{\text {th }}$ user and $\lambda$ is the carrier wavelength.

\subsection{Iterative Turbo Beamforming (ITBF)}

In the proposed ITBF, a QPSK demapper and a MAP decoder work in an iterative fashion. At each iteration for a particular OFDM symbol, BF complex weights are computed based on received pilots and soft decoded data from previous iterations. The ITBF comprises of three distinctive stages, namely Rudimentary Beamforming Stage (RBS), Iterative Beamforming Stage (IBS) and Termination Beamforming Stage (TBS).

Following from (4), the ULA output $\mathbf{V}$ for the $l^{\text {th }}$ OFDM symbol is processed by the beamformer which is given as:

$$
\mathbf{z}_{i}=\mathbf{w}_{i}^{H}\left(\mathbf{A} \mathbf{Y}^{H}+\mathbf{B}\right),
$$

where $\mathbf{z}_{i}=\left[z_{i}(0), z_{i}(1), \ldots, z_{i}(N-1)\right]$ is the weighted output of the BF corresponding to the desired user, while $\mathbf{w}_{i}=$ $\left[w_{i}(1), w_{i}(2), \ldots, w_{i}(S)\right]^{T}$ represents the beamformer's complex weights for the $i^{t h}$ iteration. When $i=0$, this stage is referred to as RBS as BF weights applied correspond to the previous OFDM symbol $\left(\mathbf{w}_{i}[l]=\mathbf{w}_{I}[l-1]\right)$. Now $\mathbf{z}_{i}$ is $\mathrm{S} / \mathrm{P}$ converted followed by FFT. This can be presented mathematically as:

$$
\widetilde{\mathbf{z}}_{i}=\mathbf{F}\left(\mathbf{w}_{i}^{H} \mathbf{A} \mathbf{Y}^{H}+\mathbf{w}_{i}^{H} \mathbf{B}\right)^{H} .
$$

When considering an AWGN channel, data sub-carriers in $\widetilde{\mathbf{z}}_{i}$ are directly de-multiplexed into $\widetilde{\mathbf{r}}_{i}^{q}$ which are passed to the QPSK demapper. For the case of wireless channel scenario, CE is performed on $\widetilde{\mathbf{z}}_{i}$ to yield $\widetilde{\mathbf{r}}_{i}$ which is then de-multiplexed into 


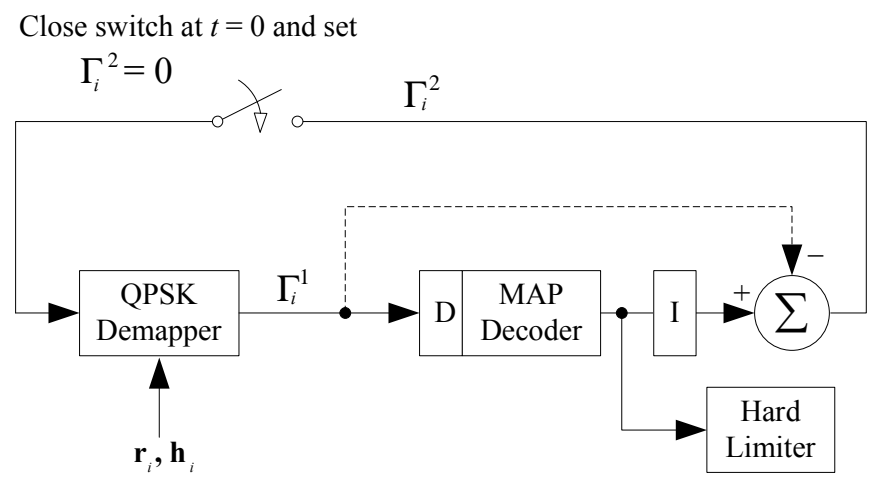

Figure 4. Information flow in Demapper/Decoder

data-sub-carriers $\widetilde{\mathbf{r}}_{i}^{q}$. We define in general the demapper task as computation of a posteriori probability (APP) given received vector $\widetilde{\mathbf{r}}_{i}^{q}$, channel estimates $\widetilde{\mathbf{h}}_{i}^{q}$ and a priori information $\Gamma_{i}^{2}$. More specifically, demapper outputs extrinsic information, or Log-Likelihood Ratio (LLR) $\Gamma_{i}^{1}$ for the $v^{t h}$ coded bit $c_{v}$ in desired user's transmitted data sequence $\widetilde{\mathbf{x}}^{q}$. This is given by:

$$
\begin{aligned}
& \Gamma_{i}^{1}\left(c_{v}\left(\widetilde{x}^{q}(n)\right)\right)=\ln \frac{\sum_{b \in U_{v}^{+}} P\left(\widetilde{x}^{q}(n)=b \mid \widetilde{r}_{i}^{q}(n), \widetilde{h}_{i}^{q}(n), \Gamma_{i}^{2}\right)}{\sum_{b \in U_{v}^{-}} P\left(\widetilde{x}^{q}(n)=b \mid \widetilde{r}_{i}^{q}(n), \widetilde{h}_{i}^{q}(n), \Gamma_{i}^{2}\right)}, \\
& P\left(\widetilde{x}^{q}(n)=b \mid \widetilde{r}_{i}^{q}(n), \widetilde{h}_{i}^{q}(n), \Gamma_{i}^{2}\right)= \\
& \frac{1}{2 \pi \sigma^{2}} \exp \left(-\frac{\left.\| \widetilde{r}_{i}^{q}(n)-\widetilde{h}_{i}^{q}(n) \widetilde{x}^{q}(n)\right) \|^{2}}{2 \sigma^{2}}\right) \prod_{u \neq v} P\left(c_{u}\left(\widetilde{x}^{q}(n)\right),\right.
\end{aligned}
$$

Fig. 4 illustrates the information flow in the demapper and the MAP decoder, where I and D are interleaving and deinterleaving blocks respectively. In (8), (9) $U_{v}^{-}$and $U_{v}^{+}$represents the constellation set that contains all the symbols whose $v^{\text {th }}$ bit is 0 and 1 respectively. $\Gamma_{i}^{1}$ is de-interleaved and passed to the MAP decoder. The MAP decoder outputs and feed backs the extrinsic information $\Gamma_{i}^{2}\left(c_{v}\left(\widetilde{x}^{q}(n)\right) . \Gamma_{i}^{2}\right.$ is interleaved and then used to compute the soft data symbols as follows:

$$
\begin{gathered}
\hat{\tilde{x}}_{i}^{q}(n)=\sum_{b \in U} b \cdot P\left(\widetilde{x}^{q}(n)=b\right), \\
P\left(\widetilde{x}^{q}(n)=b\right)=\prod_{u=1}^{\log _{2}|U|} P\left(c_{u}\left(\widetilde{x}^{q}(n)\right)\right),
\end{gathered}
$$

where $U \mid$ denotes the cardinality of the set $U$. The soft data symbols for the QPSK case can be computed by:

$$
\hat{\widetilde{x}}_{i}^{q}(n)=\frac{1}{\sqrt{2}}\left(\operatorname { t a n h } \left(\Gamma_{i}^{2}\left(c_{0}\left(\widetilde{x}^{q}(n)\right) / 2\right)+j \tanh \left(\Gamma_{i}^{2}\left(c_{1}\left(\widetilde{x}^{q}(n)\right) / 2\right)\right) .\right.\right.
$$

The conventional LMS beamformer requires the difference between transmitted and received pilots as an input (termed as the 
error vector). However with the proposed beamformer, soft data symbols and received pilots work in conjunction to perform BF. Hence the error vectors corresponding to soft data and pilots are given by:

$$
\begin{aligned}
\widetilde{\mathbf{e}}_{i}^{q} & =\hat{\mathbf{x}}_{i}^{q}-\widetilde{\mathbf{x}}^{q} . \\
\widetilde{\mathbf{e}}_{i}^{p} & =\widetilde{\mathbf{z}}_{i}^{p}-\widetilde{\mathbf{x}}^{p} .
\end{aligned}
$$

Error vectors $\widetilde{\mathbf{e}}_{i}^{q}$ and $\widetilde{\mathbf{e}}_{i}^{p}$ are mapped to known sub-carrier locations to obtain the frequency-domain Combined Error $\left(\mathrm{CE}_{f}\right)$ vector $\widetilde{\mathbf{e}}_{i}=\left[\widetilde{e}_{i}(0), \widetilde{e}_{i}(1), \ldots, \widetilde{e}_{i}(N-1)\right]^{T}$. As we employ Pre-FFT BF, $\mathrm{CE}_{f}$ is converted to the time-domain which can be presented mathematically as:

$$
\mathbf{e}_{i}=\mathbf{F}^{H} \widetilde{\mathbf{e}}_{i}
$$

$\mathbf{e}_{i}$ is used to update BF weights for the next iteration for $l^{\text {th }}$ OFDM symbol. Using (15), the LMS adaptation is given by:

$$
\mathbf{w}_{i+1}[l]=\mathbf{w}_{i}[l]+2 \mu \mathbf{V}[l] \mathbf{e}_{i}[l] .
$$

Substituting (15) into (16) we get:

$$
\mathbf{w}_{i+1}[l]=\mathbf{w}_{i}[l]+2 \mu \mathbf{V}[l] \mathbf{F}^{H} \widetilde{\mathbf{e}}_{i}[l] .
$$

It must be noted that the notation $\mathbf{w}_{i}[l]$ refers to $l^{\text {th }}$ OFDM symbol rather than the $l^{\text {th }}$ element of the weight vector $\mathbf{w}$. The new BF weights $\mathbf{w}_{i+1}$ are used for the next iteration in (6). For $1<i<I$, the process presented in (6) $-(17)$ is referred to as Iterative Beamforming Stage (IBS). The IBS continues for the desired number of iterations. In the final stage $(i=I)$ referred to as TBS, the output of MAP decoder is decoded into $\{\hat{\mathbf{o}}\}$ using hard-decision. Moreover, $\mathbf{w}_{i+1}$ computed during TBS are used for the next OFDM symbol which is given by:

$$
\mathbf{w}_{I}[l+1]=\mathbf{w}_{I}[l]+2 \mu \mathbf{V}[l] \mathbf{e}_{I}[l]
$$

where $[l]$ and $[l+1]$ in $(18)$ correspond to complex weights for consecutive OFDM symbol. $\mu$ represents the positive step size which controls the rate of convergence. The algorithm only converges [23] if:

$$
\mu_{\min } \leq \mu \leq \mu_{\max }
$$

with

$$
\mu_{\max } \leq \frac{2}{3 \operatorname{tr}(\mathbf{R})}
$$


where $\mu_{\max }$ is chosen to bound the Mean Squared Error (MSE) of the algorithm and depends on the maximum the eigenvalue of the received signal covariance matrix $\mathbf{R}$. Whereas $\mu_{\min }>0$ is chosen to provide minimum tracking capability to the algorithm. When constant $\mu$ (Fixed Step Size) is employed with LMS, $\mu$ will usually be close to $\mu_{\min }$ [4]. Rather than implementing a fixed $\mu$ which results in slower convergence, we adapt the step size at each BF iteration depending on the received signal covariance matrix $\mathbf{R}$. Hence we call this optimised LMS where $\mu$ is adapted at each iteration according to (20). The pseudo-code representation of the proposed ITBF algorithm is presented as Algorithm 1.

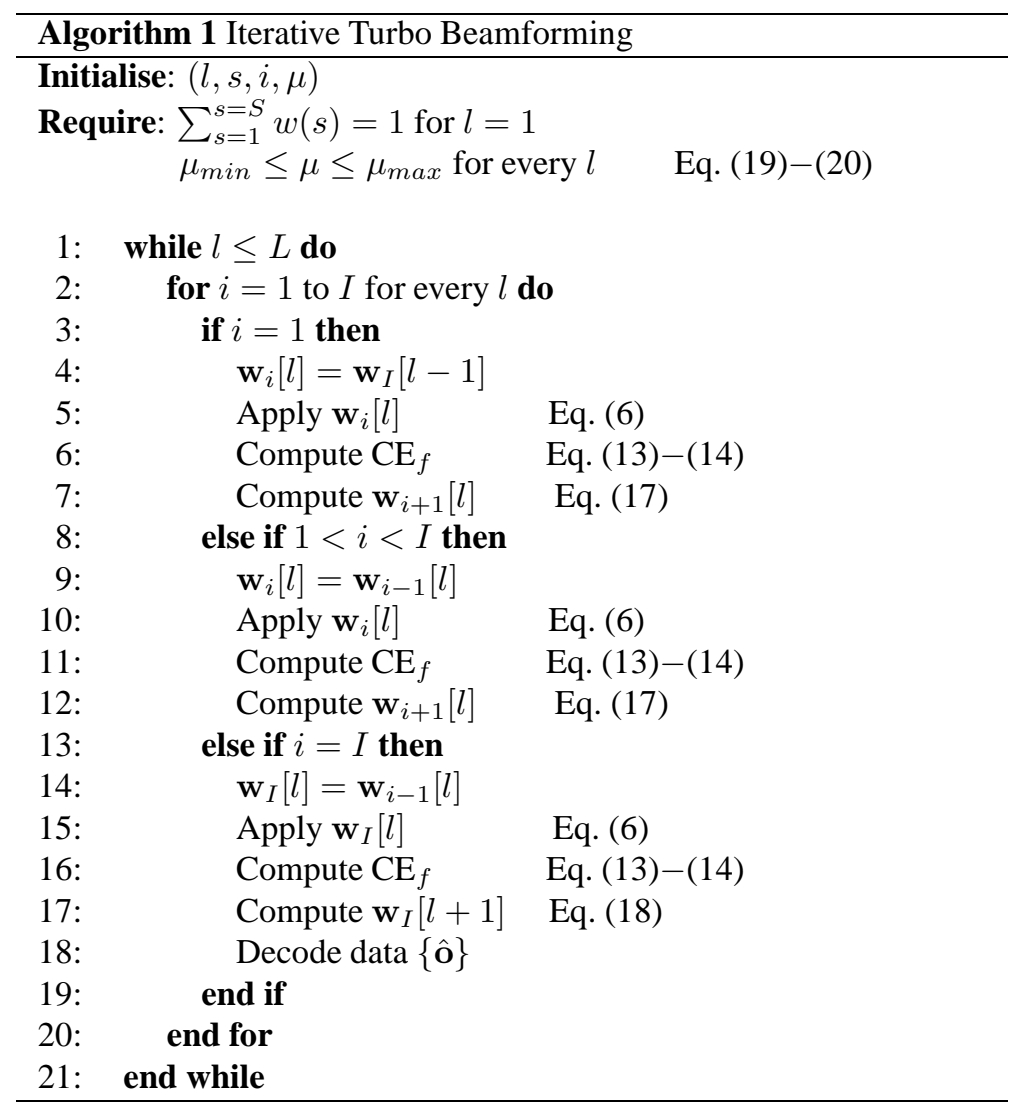

\subsection{Conventional LMS Beamforming}

In the proposed ITBF, BF weights are adapted for $I$ iterations per OFDM symbol. For the conventional LMS BF [24], complex weights are adapted once every OFDM symbol. For the conventional non-iterative LMS BF that only uses pilot sub-carriers, the weights adaptation process is given by:

$$
\mathbf{w}[l+1]=\mathbf{w}[l]+2 \mu \mathbf{V}[l] \mathbf{F}^{H} \widetilde{\mathbf{e}}[l] .
$$

Here $\mathbf{w}[l]$ and $\mathbf{w}[l+1]$ represent the beamformer's complex weights for $[l]$ and $[l+1]$ OFDM symbol. $\widetilde{\mathbf{e}}$ represents the error vector that is computed using pilot sub-carriers only. Furthermore, $\mu$ must satisfy (19) and (20) just as was the case in ITBF. 


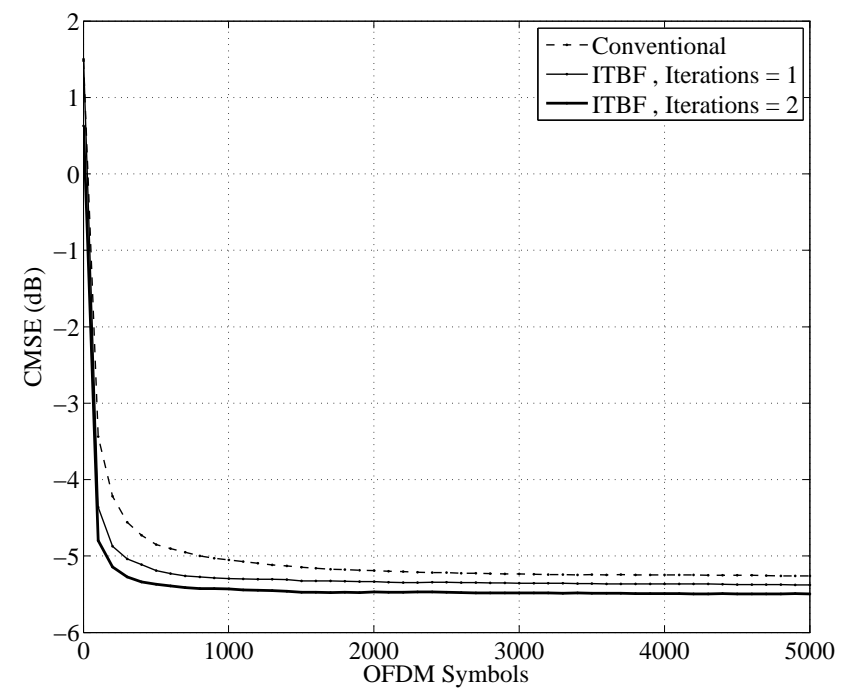

(a) Antenna elements $=2$ and $E_{b} / N_{o}=8 \mathrm{~dB}$

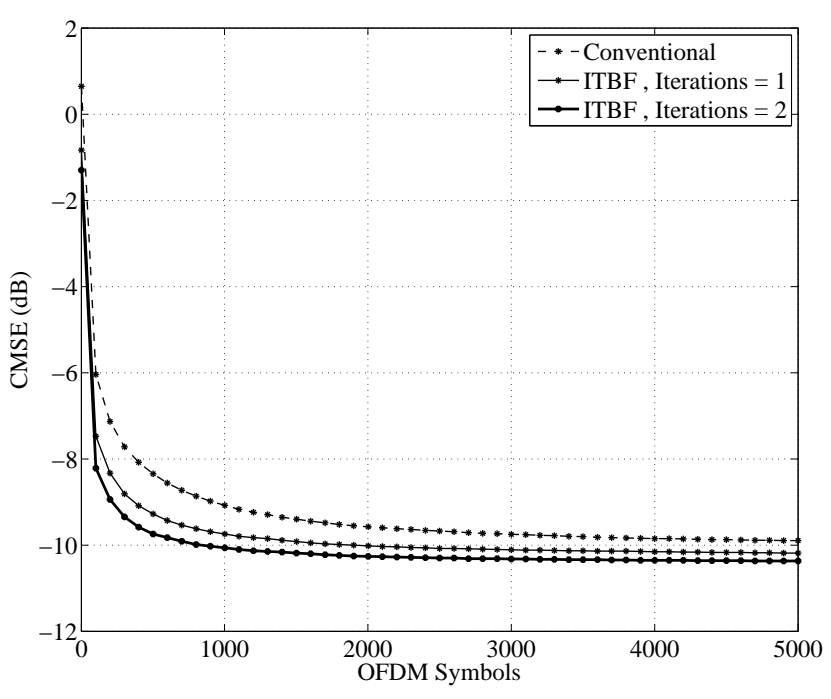

(b) Antenna elements $=4$ and $E_{b} / N_{o}=8 \mathrm{~dB}$

\section{Figure 5. Beamforming convergence in terms of Cumulative Mean Squared Error vs OFDM symbols}

If total $L$ OFDM symbols are processed with assuming $M$ multiples per OFDM symbol, then total multiplies for the non-iterative BF presented in (21) would be $M \times L$, whereas for the ITBF case this would be $(I+1) M \times L$. Hence ITBF is more complex as compared to the conventional BF approach. However, the technique would become viable if considerable gains can be achieved with minimal iterations.

\section{Simulation and Discussions}

A SIMO BICM-OFDM system with 32 sub-carriers $(N)$ having 5 pilots per OFDM symbol $\left(N_{p}\right)$ is modelled. $1 \times 2$ and $1 \times 4$ SIMO configurations are employed. In accordance with Fig. 2, one desired user was modelled at $40^{\circ}$ while interference users were located at $-70^{\circ},-35^{\circ}$ and $60^{\circ}$ azimuth respectively. A rate- $1 / 2(5,7)_{8}$ convolution encoder and random interleaver/de-interleaver are employed in an AWGN channel condition. The power per interference user at the satellite end is set to $-5 \mathrm{dBW}$ whereas power of the desired user is set to $0 \mathrm{dBW}$. The proposed ITBF (Section 3.2) is compared with conventional non-iterative adaptive LMS beamformer (Section 3.3).

Prior to presenting the results, we pause to investigate the way in which BF convergence could be studied. In literature, the convention of presenting BF convergence is the instantaneous mean error of the beamformer in terms of MSE (dB) against number of iterations passed (or in our case OFDM symbols), for instance in [24]. Another way of presenting the mean of any data in statistical theory is the Cumulative Moving Average or Running Average. Running average (or running mean) is a valuable tool and has been used in several disciplines, like digital circuits [25], economics and sociology [26], motion detection [27] to measure "learning processes". It presents the running mean of the data rather than the instantaneous mean. In case of BF, if $\omega_{l}$ is the MSE of the $l^{t h}$ OFDM symbol, then the running average (or we call it the Cumulative MSE) for $L$ 


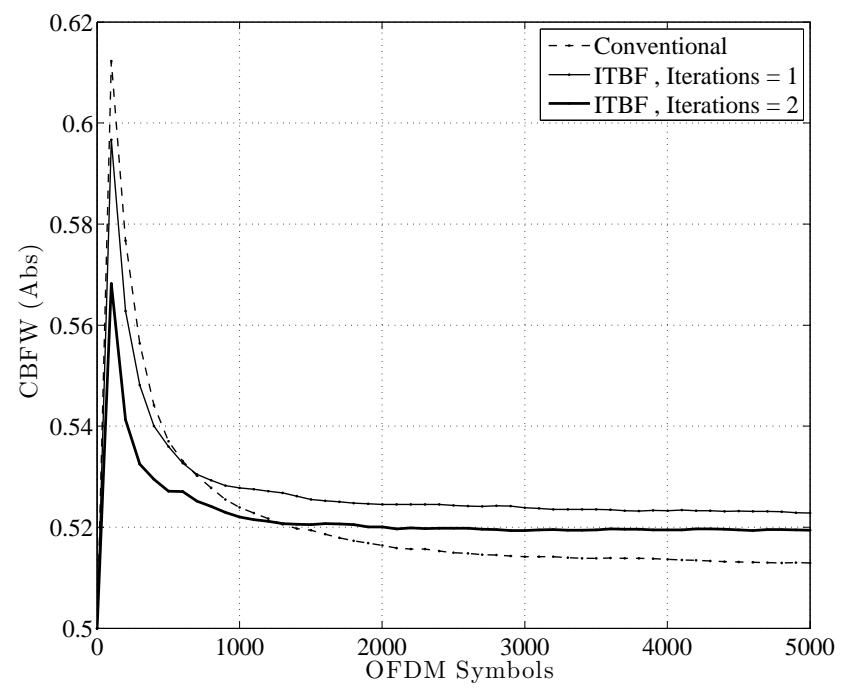

(a) Antenna elements $=2$ and $E_{b} / N_{o}=8 \mathrm{~dB}$

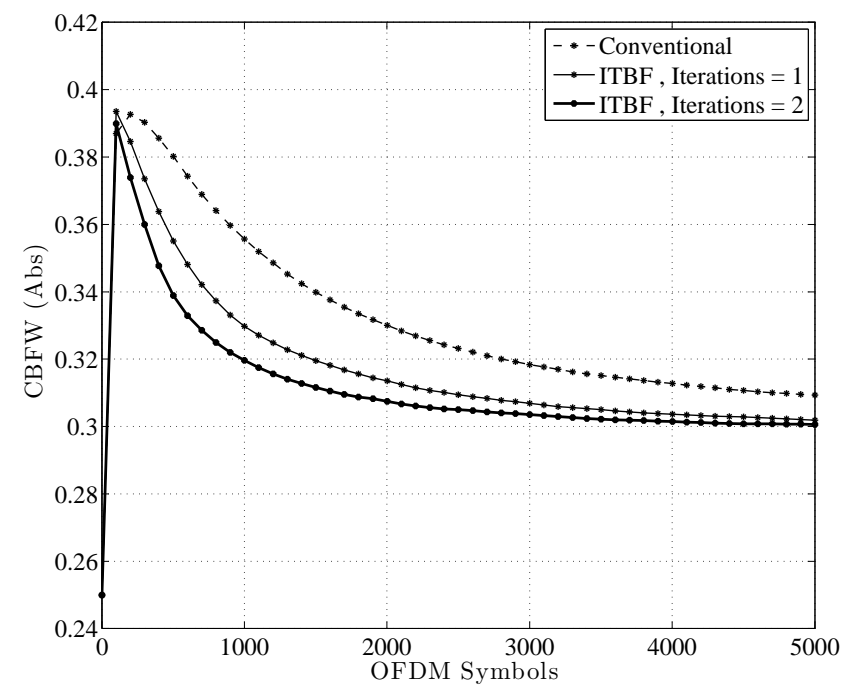

(b) Antenna elements $=4$ and $E_{b} / N_{o}=8 \mathrm{~dB}$

Figure 6. Beamforming convergence in terms of Cumulative Beamforming Weights (Abs) of one of the antenna elements vs OFDM symbols

OFDM symbols can be presented as:

$$
\mathrm{CMSE}_{L}=\left[\omega_{1}, \frac{\omega_{1}+\omega_{2}}{2}, \frac{\omega_{1}+\omega_{2}+\omega_{3}}{3}, \cdots, \frac{\sum_{l}^{L} \omega_{l}}{L}\right]
$$

Now using the running mean as defined in (22), in Fig. 5(a) we analyse the BF performance in terms of Cumulative Mean Squared Error (CMSE) at pilot locations against OFDM symbols for the case of 2 antenna element configuration. In other words this figure presents the transient and the steady state behaviour of BF in terms of prediction error for a specific $E_{b} / N_{o}$ level. We can see that ITBF converges faster as well as attains a lower CMSE as compared to the conventional case. Moreover, within the ITBF framework, further improvement in the minimum CMSE achieved as well as speed of convergence is observed with increased number of iterations $(i)$. Effectively, iterative BF translates to better CCI mitigation as well as shorter transient state time.

Now if we increase the number of antenna elements to 4, we can observe in Fig. 5(b) that a much lower CMSE is obtained in all considered schemes as compared to $S=2$ case. However, convergence speed in case of proposed ITBF is superior as compared to the conventional case. We can also observe further reduction in minimum CMSE achieved as well as transient time with increased number of iterations. To compare the case of 2 and 4 antenna elements, we present a snapshot of the CMSE for the two configurations at $l=300$ in Fig. 7. We can observe an increasing trend of improved CMSE as the iterations increase when BF has processed 300 OFDM symbols irrespective of the number of antenna elements employed. Moreover, we can observe a reduction in CMSE for $S=4$, with ITBF showing superior performance. In conclusion, there 


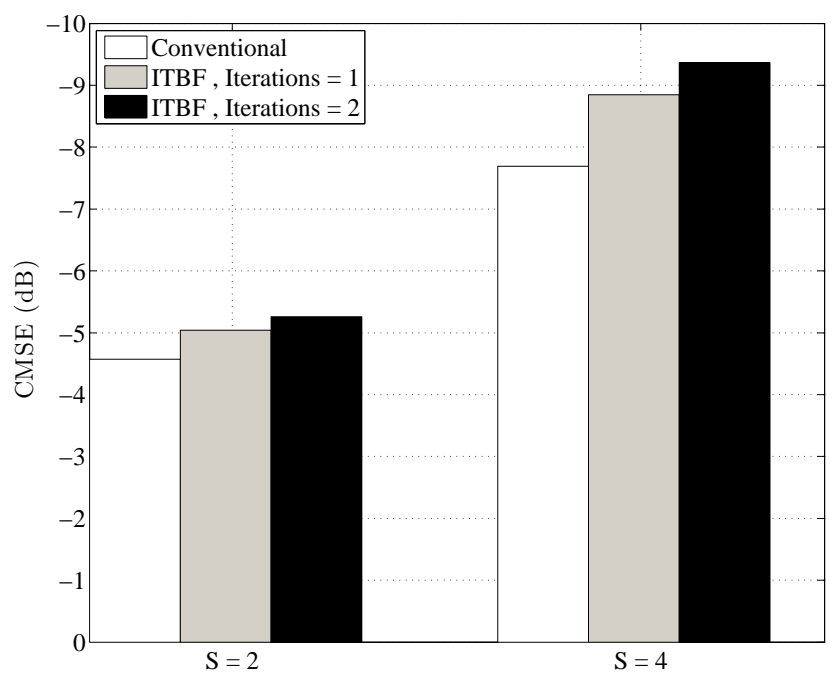

Figure 7. Snapshot of Cumulative Mean Squared Error at $300^{\text {th }}$ OFDM symbol, antenna elements $=2 \&$ 4 and $E_{b} / N_{o}=8 \mathbf{d B}$.

are two major reasons for aforementioned trend 1) interference suppression improves with more antenna elements and 2) the 2 antenna configuration cannot simultaneously steer nulls in the direction of all 3 interference sources.

Another perspective of BF performance is how the BF complex weights adapt w.r.t time. Fig. 6(a) presents the Cumulative Beamforming Weights (CBFW) for $S=2$ configuration for only one of the antenna element, and hence a scalar plot. Just as was the case with CMSE in Fig. 5, the CBFW presents the running average of BF weights. The cumulative form of weights is used to present the averaged effect. It is evident that BF weights for the conventional case exhibit highest latency in terms of convergence. With one iteration, the weights convergence speed is enhanced which is further improved with 2 iterations. For the case of $S=4$, the BF weights adaptation is shown in Fig. 6(b). We observe a similar trend to the case with 2 antenna elements. This further validate CMSE results as well as the performance advantage of the ITBF approach.

The CMSE depicted in Fig. 5 and 7 demonstrate how ITBF improves BF convergence as well as prediction error. However since CMSE is only evaluated at pilot locations, whereas BF takes places using both pilots and data, results do not depict the true performance advantages of ITBF. Therefore it is imperative to analyse the error rate performance of the ITBF as compared to the conventional non-iterative BF. Hence we look at the Bit Error Rate (BER) in Fig. 8 show the performance advantages of ITBF. It is evident from the BER result that ITBF outperforms the conventional case irrespective of $E_{b} / N_{o}$. With only one iteration, the ITBF provides a BER Gain $($ BERG) of $\approx 1.5 \mathrm{~dB}$ with 2 antenna elements and increases to $\approx 2.5$ $\mathrm{dB}$ when 4 antenna elements are employed. Furthermore, at a worst case scenario of $E_{b} / N_{o}=0 \mathrm{~dB}$, the proposed approach exhibits far superior performance compared to the conventional case.

Finally, we look at an end-to-end HTSMS scenario and based on the BER results compare the throughput of conventional system against one employing ITBF. We also consider Carrier Frequency Offset (CFO) in the system which arises Inter 


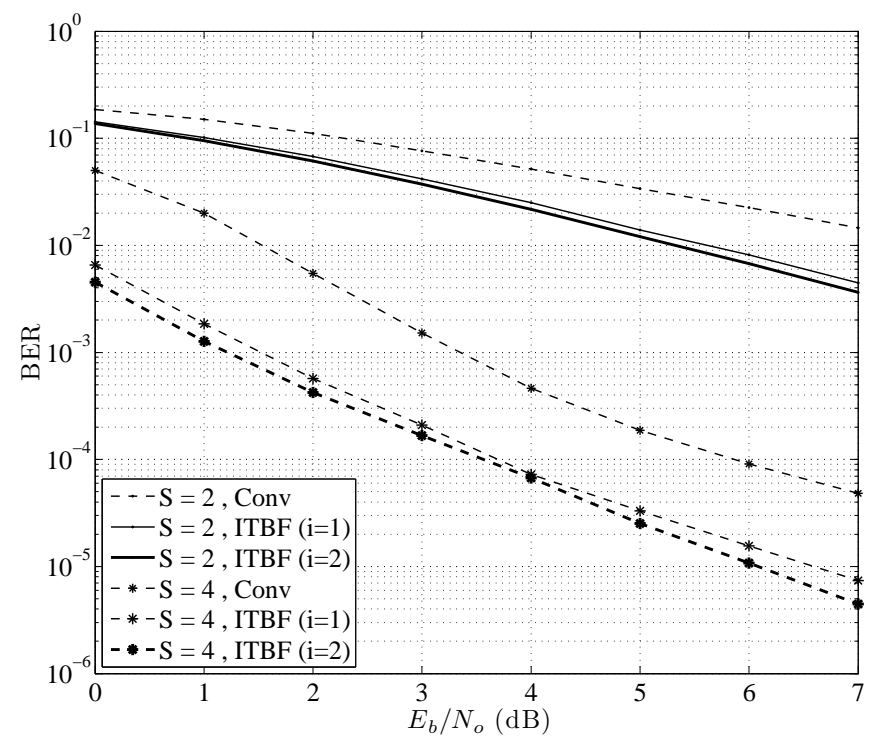

Figure 8. Bit Error Rate vs desired user $E_{b} / N_{o}$, antenna elements $=2 \& 4$.

Carrier Interference (ICI) and analyse its impact on the system's throughout. Focusing on the return link depicted in Fig. 1, we compute throughput while considering typical MSS parameters as depicted in Table 1. Our throughput results are based on a realistic assumption that in the return link, Satellite-to-Hub link has the lowest available $C / N_{o}$. Based on the parameters and BER results, the throughput for conventional and ITBF system is plotted in Fig. 9 for the case of No CFO and with CFO with different target BER. We can see in Fig. 9(a) that due to BERG of the ITBF approach, we have much higher throughput as compared to the conventional system. Furthermore, when CFO is introduced in the system, this causes an increase in ICI and hence we have reduced throughput as depicted in Fig. 9(b). In general we can observe that a throughput increase of upto $41 \%$ can be achieved with ITBF approach in the case of 4 antenna element configuration.

\begin{tabular}{lr}
\hline \multicolumn{1}{c}{ Carrier Data } \\
\hline Code Rate & $\frac{1}{2}$ \\
Filler Roll-Off & $25 \%$ \\
Modulation & QPSK \\
Transmission overhead & $10 \%$ \\
\hline \multicolumn{2}{c}{ Downlink Data } \\
\hline EIRP per carrier & $35 \mathrm{~dB}$ \\
Free Space Loss & $195 \mathrm{~dB}$ \\
Pointing Loss & $0.5 \mathrm{~dB}$ \\
Rain Loss & $6 \mathrm{~dB}$ \\
Earth Station G/T & $35 \mathrm{~dB}$ \\
System Temperature & $120^{\circ} \mathrm{K}$ \\
Implementation Margin & $3 \mathrm{~dB}$ \\
Intermodulation Interference & $1 \mathrm{~dB}$ \\
ICI due to CFO & $1 \mathrm{~dB}$ \\
\hline
\end{tabular}

Table 1. MSS Parameters 


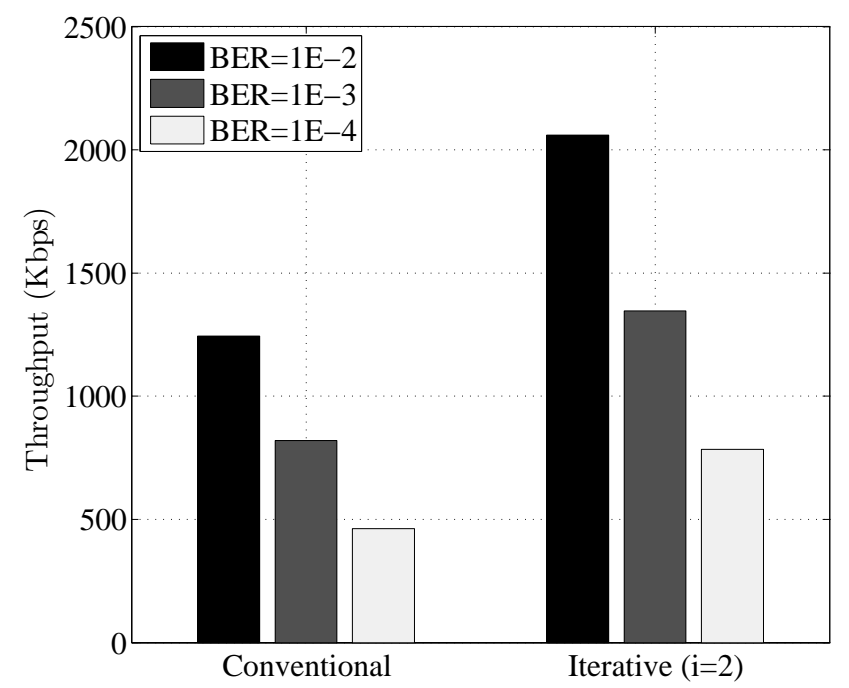

(a) Throughput with no ICI

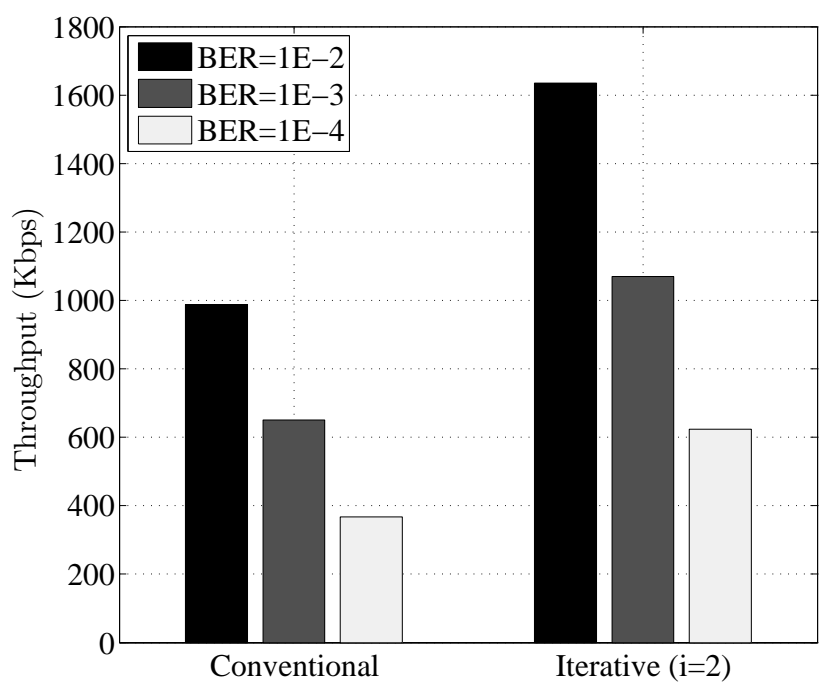

(b) Throughput with ICI due to CFO

Figure 9. Throughput comparison of conventional system against proposed ITBF with and without CFO, $S=4$

\section{Conclusions}

In this paper we have proposed a novel Iterative Beamforming (ITBF) algorithm comprising of three distinctive stages namely; Rudimentary Beamforming Stage (RBS), Iterative Beamforming Stage (IBS) and Termination Beamforming Stage (TBS). We then compared the performance of the proposed approach to the conventional non-iterative BF case and report considerable gain in terms of system performance in high interference level scenarios. As we increase the number of iterations for the ITBF, we observe improved system performance. Even in worse case scenarios such as at $E_{b} / N_{o}=0 \mathrm{~dB}$, the proposed algorithm outperforms the conventional case providing significant gain. Although iterative receiver architectures are complex, improved BER is observed with only one iterations hence making it a practical possibility. We also investigate the throughput of an end-to-end HTSMS scenario incorporating conventional and ITBF approaches and show that ITBF provides a promising gain. The ITBF has been proposed for a mobile-satellite scenario in which spectrum is shared between two regions, however the approach is equally applicable to terrestrial systems.

\section{References}

[1] A. Khan, M. Imran, and B. Evans, "Adaptive beamforming for OFDM based hybrid mobile satellite system," 27th ICSSC 2009, American Institute of Aeronautics and Astronautics, June 2009.

[2] D. Slock, "On the convergence behavior of the LMS and the Normalized LMS algorithms," Signal Processing, IEEE Transactions on, vol. 41, no. 9, pp. 2811 - 2825, Sep. 1993. 
[3] S. Douglas, “A family of Normalized LMS algorithms," Signal Processing Letters, IEEE, vol. 1, no. 3, pp. 49 - 51, Mar. 1994.

[4] R. Kwong and E. Johnston, "A variable step size LMS algorithm," Signal Processing, IEEE Transactions on, vol. 40, no. 7 , pp. 1633 - 1642, July 1992 .

[5] A. Khan, M. Imran, and B. Evans, "Preamble based adaptive beamformer for hybrid terrestrial-satellite mobile system," 28th ICSSC 2010, American Institute of Aeronautics and Astronautics, Sep. 2010.

[6] M. Lei, P. Zhang, H. Harada, and H. Wakana, "LMS adaptive beamforming based on Pre-FFT combining for ultra high-data-rate OFDM system," in Vehicular Technology Conference, 2004. VTC2004-Fall. 2004 IEEE 60th, vol. 5, Sep. 2004 , pp. $3664-3668$.

[7] A. H. Khan, M. A. Imran, and B. G. Evans, "OFDM based adaptive beamforming for hybrid terrestrial-satellite mobile system with pilot reallocation," Satellite and Space Communications, 2009. IWSSC 2009. International Workshop on, pp. $201-205$, Sep. 2009.

[8] C. Berrou, A. Glavieux, and P. Titmajshima, "Near shannon limit error-correction coding and decoding: Turbo codes," ICC, Geneva, Switzerland, pp. 1064 - 1090, May 1993.

[9] M. Sellathurai and S. Haykin, "A nonlinear iterative beamforming technique for wireless communications," Signals, Systems, and Computers, 1999. Conference Record of the Thirty-Third Asilomar Conference on, vol. 2, pp. 957 - 961, Oct. 1999.

[10] J. G. Foschini, "Layered space-time architecture for wireless communication in a fading environment when using multi element antennas," Bell Labs Tech. J, vol. 2, pp. 41 - 59, Autumn 1996.

[11] M. Reed and P. Alexander, "Iterative multiuser detection using antenna arrays and FEC on multipath channels," Selected Areas in Communications, IEEE Journal on, vol. 17, no. 12, pp. 2082 - 2089, Dec. 1999.

[12] D. Mottier and L. Brunel, "A low complexity turbo adaptive interference cancellation using antenna arrays for WCDMA,” Vehicular Technology Conference, 2001. VTC 2001 Fall. IEEE VTS 54th, vol. 3, pp. 1644 - 1648, Oct. 2001.

[13] T. Hunziker, T. Aono, and T. Ohira, "An iterative beamforming and decoding procedure for wireless networks with uncoordinated channel access," Communications Letters, IEEE, vol. 8, no. 4, pp. 256 - 258, April 2004.

[14] _ , "An iterative beamforming technique for OFDM-based wireless networks with uncoordinated channel access," Communications, 2004 IEEE International Conference on, vol. 5, pp. 2751 - 2756, June 2004. 
[15] A. Pollok, W. Cowley, and N. Letzepis, "Symbol-wise beamforming for MIMO-OFDM transceivers in the presence of co-channel interference and spatial correlation," Wireless Communications, IEEE Transactions on, vol. 8, no. 12, pp. 5755 - 5760, Dec. 2009.

[16] M. Sandell and V. Ponnampalam, "Smooth beamforming for OFDM," Wireless Communications, IEEE Transactions on, vol. 8, no. 3, pp. 1133 - 1138, March 2009.

[17] M. Zhao, Z. Shi, and M. Reed, "Iterative turbo channel estimation for OFDM system over rapid dispersive fading channel," Wireless Communications, IEEE Transactions on, vol. 7, no. 8, pp. 3174 - 3184, Aug. 2008.

[18] H. Matsuoka and H. Shoki, "Comparison of Pre-FFT and Post-FFT processing adaptive arrays for OFDM systems in the presence of co-channel interference," in Proc. 14th IEEE on Personal, Indoor and Mobile Radio Communications PIMRC, vol. 2, Sep. 2003, pp. $1603-1607$.

[19] Y. Sun and H. Matsuoka, "A novel adaptive antenna architecture - subcarrier clustering for high-speed OFDM systems in presence of rich co-channel interference," Vehicular Technology Conference, 2002. VTC Spring 2002. IEEE 55th, vol. 3, pp. 1564 - 1568, Aug. 2002.

[20] S. Li, D. Huang, K. Letaief, and Z. Zhou, "Multi-stage beamforming for coded OFDM with multiple transmit and multiple receive antennas," Wireless Communications, IEEE Transactions on, vol. 6, no. 3, pp. 959 - 969, Mar. 2007.

[21] M. Luke and R. Reggiannini, "Carrier frequency acquisition and tracking for OFDM systems," Communications, IEEE Transactions on, vol. 44, no. 11, pp. 1590 - 1598, Mar 1996.

[22] M. Luise, M. Marselli, and R. Reggiannini, "Low-complexity blind carrier frequency recovery for OFDM signals over frequency-selective radio channels," Communications, IEEE Transactions on, vol. 50, no. 7, pp. 1182 - 1188 , Jul 2002.

[23] A. Feuer and E. Weinstein, "Convergence analysis of LMS filters with uncorrelated gaussian data," Acoustics, Speech and Signal Processing, IEEE Transactions on, vol. 33, no. 1, pp. 222 - 230, Feb. 1985.

[24] C. K. Kim, K. Lee, and Y. S. Cho, "Adaptive beamforming algorithm for OFDM systems with antenna arrays," IEEE Transactions on Consumer Electronics, vol. 46, no. 4, pp. 1052 - 1058, Nov. 2000.

[25] J. Macias and A. Exposito, "Computation of running averages," Circuits and Systems II: Express Briefs, IEEE Transactions on, vol. 53, no. 10, pp. 1098 - 1102, 2006.

[26] K. Kwon and R. J. Kish, "A comparative study of technical trading strategies and return predictability: An extension of using NYSE and NASDAQ indices," Q. Rev. Econ. Finance, vol. 42, no. 3, pp. 611 - 631, 2002. 
[27] Z. Yi and F. Liangzhong;, "Moving object detection based on running average background and temporal difference," Intelligent Systems and Knowledge Engineering (ISKE), 2010 International Conference on, pp. 270 - 272, 2010. 